This item was submitted to Loughborough's Research Repository by the author.

Items in Figshare are protected by copyright, with all rights reserved, unless otherwise indicated.

\title{
Miniaturization of circular patch microstrip antenna using an arc projection
}

PLEASE CITE THE PUBLISHED VERSION

http://dx.doi.org/10.1109/LAWP.2016.2586749

\section{PUBLISHER}

Institute of Electrical and Electronics Engineers (IEEE)

VERSION

VoR (Version of Record)

\section{PUBLISHER STATEMENT}

This work is made available according to the conditions of the Creative Commons Attribution 3.0 Unported (CC BY 3.0) licence. Full details of this licence are available at: http://creativecommons.org/licenses/by/3.0/

\section{LICENCE}

CC BY 3.0

\section{REPOSITORY RECORD}

Motevasselian, Alireza, and W.G. Whittow. 2019. "Miniaturization of Circular Patch Microstrip Antenna Using an Arc Projection". figshare. https://hdl.handle.net/2134/21931. 


\title{
Miniaturization of a Circular Patch Microstrip Antenna Using an Arc Projection
}

\author{
Alireza Motevasselian, Member, IEEE, and William G. Whittow, Senior Member, IEEE
}

\begin{abstract}
A semicircle arc projection is introduced to miniaturize the patch in circular patch microstrip antennas. The idea has been inspired from the effectiveness of a cuboid ridge in size reduction of a rectangular patch microstrip antenna. A prototype of the proposed antenna together with a prototype of a conventional circular patch antenna has been fabricated and measured. The two antennas are compared, and the results are presented and discussed. A parametric study is also carried out on the characteristics of the arc projection.
\end{abstract}

Index Terms-Arc projection, circular patch, microstrip antenna, miniaturization, nonuniform patch, size reduction.

\section{INTRODUCTION}

$\mathbf{T}$ HE permanent demand for low-cost compact antennas that can be integrated into the electronic and radio-frequency components has made patch antennas an attractive alternative in modern wireless communication systems. Nevertheless, the dimensions of the patch for an antenna operating in its fundamental mode may be too large for some practical applications. The rectangular patch has a resonant dimension in order of half a wavelength. Almost the same conditions exist for the diameter of a circular-shaped patch in terms of wavelength [1].

Several techniques have been proposed and reported in the literature to achieve patch size miniaturization. The most wellknown techniques consist of using shorting wall [2], [3], shorting pin [4], high-dielectric-constant material [5], [6], folded patch [7], loading the patch with an inductive notch [8], [9], and irregular ground plane [10].

Recently, an effective method for the miniaturization of the rectangular patch antenna has been reported in [11] that is based on inductively loading the patch using a cuboid ridge. In this letter, the idea of using nonuniform patches has been utilized to miniaturize circular patch antennas. The cuboid ridge in the rectangular patch antenna is appropriately mapped into the circular patch.

In this letter, it is shown that the arc projection provides a significant reduction in the patch area as compared to a conven-

Manuscript received January 8, 2016; accepted June 28, 2016. Date of publication July 14, 2016; date of current version March 20, 2017. This work was supported by Engineering and Physical Science Research Council (EPSRC) under EP/K011383/1 Grant. In line with EPSRC guidelines, this work is available Open Access.

A. Motevasselian is with LEAX Arkivator Telecom, 41104 Gothenburg, Sweden (e-mail: alireza.motevasselian@kth.se).

W. G. Whittow is with the School of Electronic, Electrical and System Engineering, Loughborough University, Loughborough LE11 3TU, U.K. (e-mail: w.g.whittow@lboro.ac.uk).

Color versions of one or more of the figures in this letter are available online at http://ieeexplore.ieee.org.

Digital Object Identifier 10.1109/LAWP.2016.2586749

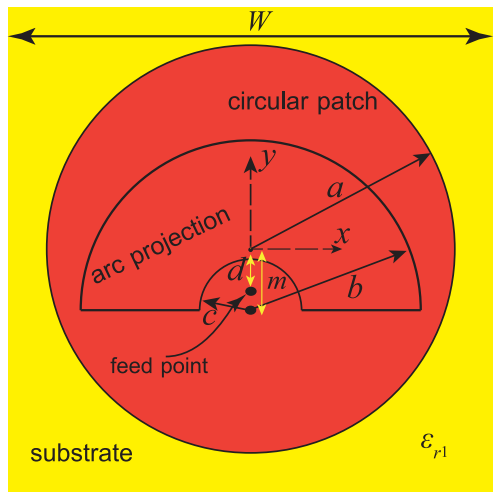

(a)

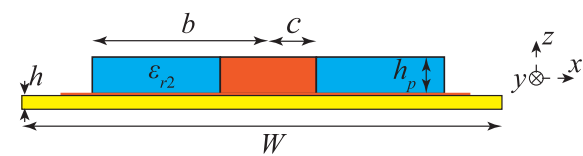

(b)

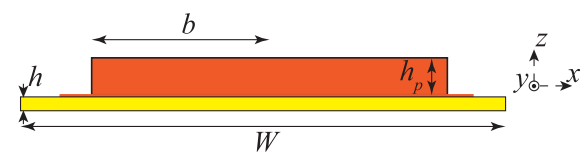

(c)

Fig. 1. Configuration of the proposed antenna with the arc projection. (a) Top view. (b) Front-side view. (c) Back-side view.

tional circular patch microstrip antenna operating in the same frequency. In the proposed configuration, the size of the patch can be adjusted using two extra parameters: the projection height and the arc width. Hence, the proposed antenna provides the designer with a higher degree of design flexibility. The simulation results indicate no substantial degradation in the gain of the antenna with an arc projection as compared to a conventional patch antenna on a similar substrate and operating in the same frequency band.

\section{CONFiguration OF THE ANTENNA}

The geometry of the proposed antenna is illustrated in Fig. 1. The configuration consists of a grounded square dielectric substrate of lateral dimension $W$, thickness $h$, and dielectric constant $\varepsilon_{r 1}$. An electrically conducting circular patch of radius $a$ is concentrically located on the radiating face of the dielectric substrate. An annular $180^{\circ}$ arc such that its center has an offset $m$ with respect to the center of the circular patch is removed from the patch. A dielectric arc projection of height $h_{p}$ and 


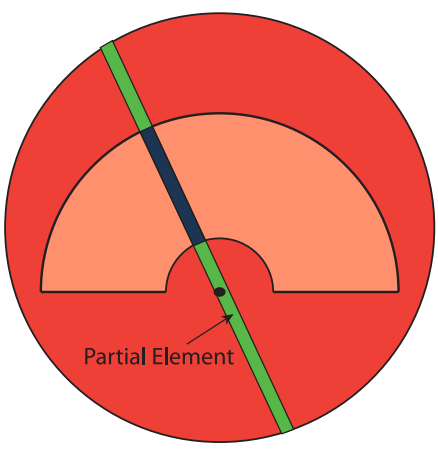

Fig. 2. Partial rectangular element on the proposed antenna structure.

dielectric constant $\varepsilon_{r 2}$ is placed at this removed area. The outer and inner radii of the arc projection are $b$ and $c$, respectively. The top face and the curved inner and outer sides of the projection are covered with electrically conducting layers that connect the top conducting face of the arc to the patch. The antenna is fed with a coaxial probe at a point, which has an offset $d$ to the center of the patch. The dielectric materials used for the substrate have been considered lossless.

As mentioned in Section I, the idea of arc projection inspired from the impressive effect of a cuboid ridge on the miniaturization of the rectangular patch antenna with its theoretical explanation has been explained in [11] in detail. The role of cuboid ridge is basically like a short stub in transmission line model of the antenna that loads the antenna inductively and reduces the resonance frequency.

To efficiently project the idea of flange ridge on the miniaturization of a circular patch, the rectangular structure is cleverly mapped to a circular one. Based on an engineering estimation, a circular patch can be created by $180^{\circ}$ sweep integration of infinitesimally wide diagonal rectangular patches centered at the feeding point. If a rectangular flange exists on each of the rectangular partial elements, the resulting structure would be the proposed antenna, as shown in Fig. 2. The flange on the partial element reduces the length of the each partial element based on the theory explained in [11], and this leads to a reduction of radius in the resulting circular structure.

\section{RESUlTS AND DISCUSSION}

A sample antenna of the proposed type is designed, fabricated, and measured. This antenna is compared to a conventional circular patch antenna operating at the same frequency band. Both of the antennas are etched on a $0.8-\mathrm{mm}$-thick dielectric substrate of dielectric constant $\varepsilon_{r 1}=2.2$. The metallic surface area under the arc projection is removed, and a dielectric arc of thickness $1.9 \mathrm{~mm}$ is placed on this area. The arc projection is made of a plastic material by means of a 3-D printing machine. This plastic material has a dielectric constant of $\varepsilon_{r 2}=2.94$ and loss tangent of $\tan \delta=0.027$, measured at $f=2.4 \mathrm{GHz}$ that are measured with a split-post dielectric resonator.

The arc projection has been fixed at its location using a few small drops of super glue. The top and curved side walls of the arc projection are covered with pieces of copper tape with conductive adhesive, as shown in Fig. 3.

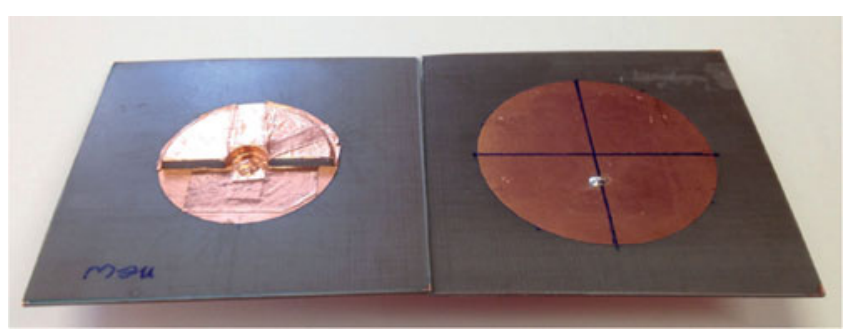

Fig. 3. Comparison of the size of the fabricated prototypes of a conventional circular patch antenna and a circular patch with an arc projection both operating at the same frequency band in their fundamental mode.

TABLE I

DESIGN PARAMETERS FOR THE FABRICATED PROTOTYPE

\begin{tabular}{lccccccccc}
\hline \hline$a$ & $b$ & $c$ & $d$ & $h$ & $m$ & $h_{p}$ & $W$ & $\varepsilon_{r 1}$ & $\varepsilon_{r 2}$ \\
18 & \multirow{2}{*}{17} & 4 & 2 & 0.8 & 2.6 & 2 & 70 & 2.2 & 2.94 \\
\hline \hline
\end{tabular}

The dimensions are in millimeters.

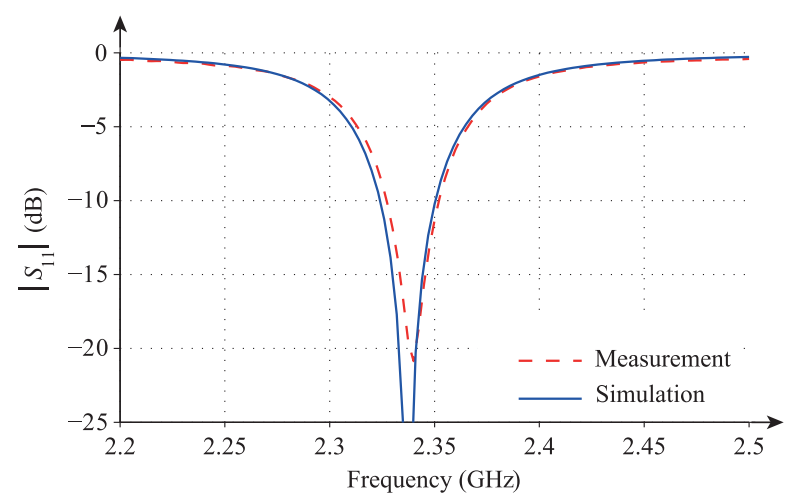

(a)

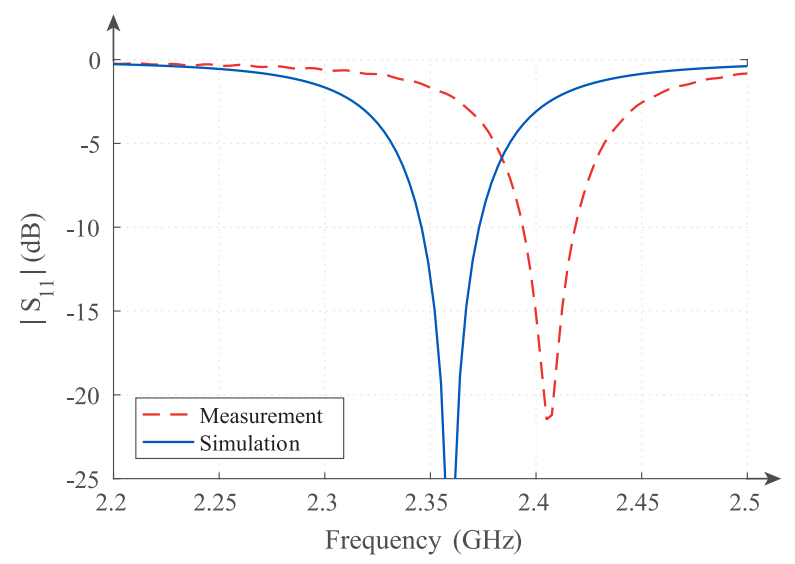

(b)

Fig. 4. Simulation and measured results for reflection coefficient.

The antenna is fed by a coaxial line at a point with a distance $2 \mathrm{~mm}$ from the center of the patch. The center of the arc projection is located with a distance $2.6 \mathrm{~mm}$ from the center of the patch. The value for $m$ has been chosen as small as the prototype fabrication, and feeding is possible and simple. The design parameters are listed in Table I.

The measured and simulated $\left|S_{11}\right|$ response of the antenna as a function of frequency is shown in Fig. 4. It is observed that the antenna resonates at $f=2.34 \mathrm{GHz}$. A good agreement is observed between the measured and simulated results for the 


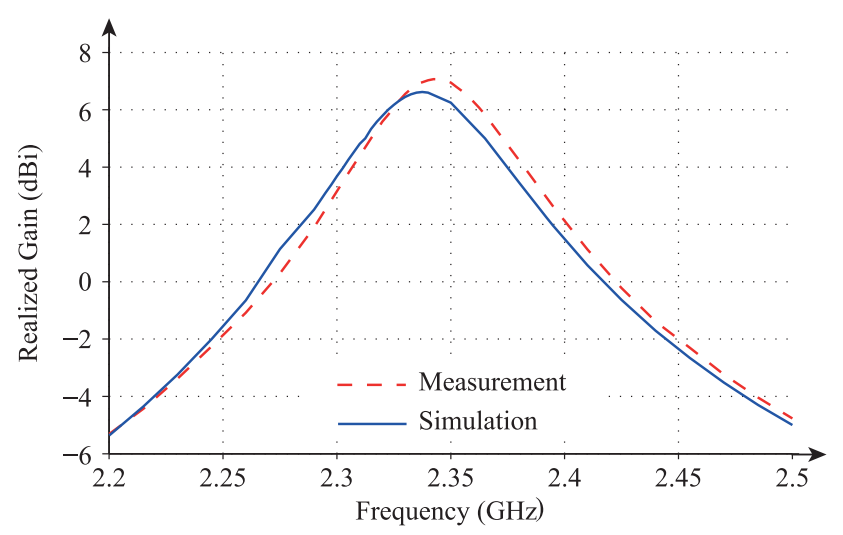

Fig. 5. Simulation and measured results for realized gain as a function of frequency.

reflection coefficient. It should be noted that the dielectric materials constructing the substrate were considered to be lossless in the simulated structure.

In order to have a comparison reference, a conventional flat circular patch microstrip antenna operating in the same frequency band is also fabricated and measured. For a visual comparison of the patch sizes, the two fabricated prototypes are depicted in Fig. 3. The patch radius for the conventional circular patch microstrip antenna is $24 \mathrm{~mm}$, while it is $18 \mathrm{~mm}$ for the antenna with the arc projection. This shows that $21 \%$ reduction in the patch radius that gives a $37 \%$ reduction in the area under patch has been achieved by the presented approach for this sample design. It should be noted that depending on the application and the available size for the antenna, the size reduction rate can be increased by changing the height or width of the arc projection. This provides the designer with at least two more degrees of freedom for the antenna design. All simulation results in this letter have been achieved from the CST Microwave Studio solver.

The measured and simulated realized gains of the antenna with the arc projection versus frequency are shown in Fig. 5. The peak gain is 7 and $6.7 \mathrm{dBi}$ for the measurement and simulation, respectively. The measured and simulated results are in agreement. The realized gain for the fabricated conventional circular patch antenna is 8.4 and $7.8 \mathrm{dBi}$, respectively, in the measurement and simulation. The gain of the proposed antenna with the arc projection is, respectively, lower than the gain of the conventional antenna. This is due to the smaller size of the patch and also the amount of lossy copper tape used in the fabrication of the arc projection. Since the gain of the antenna is proportional to its physical aperture size and the physical aperture size of the circular patch antenna is proportional to its radius, we have

$$
\frac{G_{a}}{G_{c}} \approx \frac{R_{a}}{R_{c}} \frac{E_{a}}{E_{c}}
$$

where $G_{a}, G_{c}, R_{a}, R_{c}$, and $E_{a}$ and $E_{c}$ are the gain, radius, and efficiency of the antenna with arc projection and conventional patch, respectively. Inserting the achieved values from simulation and measurement in (1), the ratio of efficiencies of two antennas would stay in the interval of $0.97<\frac{E_{a}}{E_{c}}<1.03$. It
TABLE II

Comparison of Prototypes of the Conventional Patch AND the Patch WITH AN ARC PROJECTION

\begin{tabular}{lcc}
\hline \hline & Flat patch & Patch with arc \\
\hline Radius of the patch $(\mathrm{mm})$ & 24 & 18 \\
CST $f_{r}(\mathrm{GHz})$ & 2.36 & 2.34 \\
Measured $f_{r}(\mathrm{GHz})$ & 2.41 & 2.34 \\
CST Realized Gain $(\mathrm{dBi})$ & 7.8 & 6.7 \\
Measured Peak Realized Gain $(\mathrm{dBi})$ & 8.4 & 7.1 \\
\hline \hline
\end{tabular}

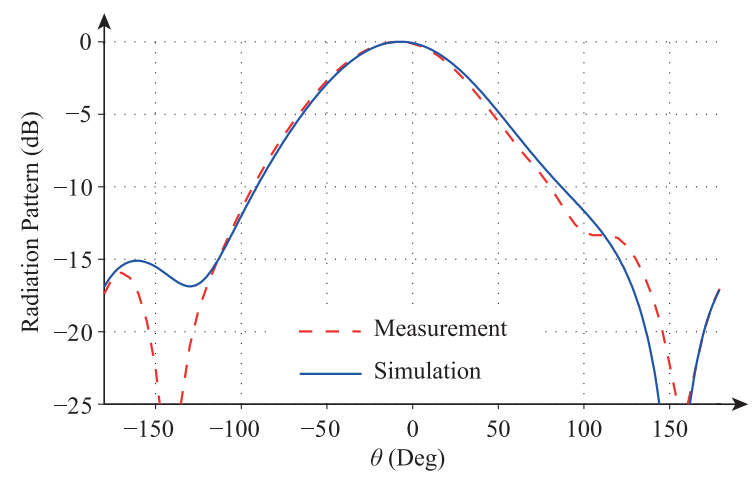

Fig. 6. Simulation and measured results for radiation pattern at $2.34 \mathrm{GHz}$, at $\phi=90^{\circ}$ cut plane.

means that the antenna with the arc projection provides similar efficiency as the conventional patch antenna.

The different value of the gain in the measurement result as compared to corresponding simulation results can be due to the measurement uncertainty of the anechoic chamber. A summary of the measurement and simulation results of the two antennas is presented in Table II.

The measured and simulated $E$-plane radiation patterns of the fabricated antenna with the arc projection are plotted in Fig. 6 at $2.34 \mathrm{GHz}$. The measured and simulation radiation patterns are in agreement. It is observed that the antenna produces a fairly symmetric broadside radiation pattern.

The antenna has been simulated using CST Microwave Studio and measured at Loughborough University's anechoic chamber.

\section{PARAmetric Study of the Arc Projection}

The volume of the arc projection determines the level of miniaturization of the patch area. To achieve a smaller patch size, one can increase either the arc width, $(b-c)$, or the projection height, $h_{p}$, depending on the designer preferences and the practical limitations and requirements of the application. In this section, a simulation-based investigation on the dimensions of the arc projection is presented.

The variation of the resonant frequency as a function of projection height with the arc thickness of $(b-c)=13 \mathrm{~mm}$ for circular patch antennas with two different values of substrate thickness and dielectric constant of $\varepsilon_{r 1}=2.2$ is shown in Fig. 7. The characteristics of the material in the arc projection were considered the same as the fabricated prototype. As observed in the figure, the rate of the resonant frequency reduction is more for the smaller value of the projection height. As the projection 


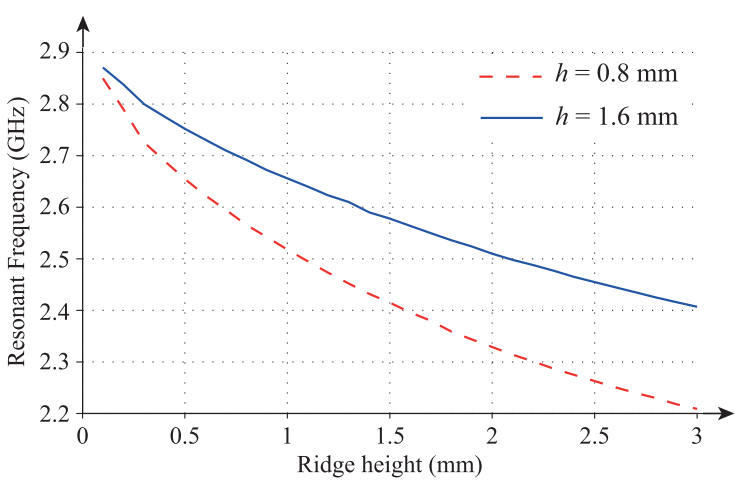

Fig. 7. Simulation results for variation of the resonant frequency with the height of the projection $\left(h_{r}\right)$ for two different values of substrate thickness.

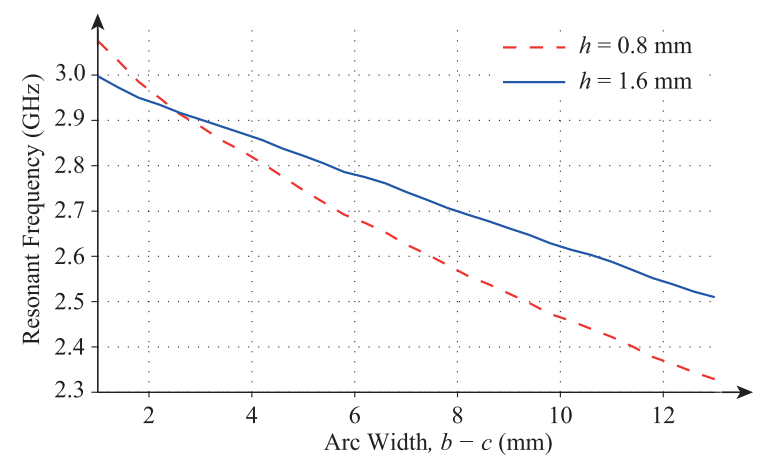

Fig. 8. Simulation results for variation of the resonant frequency with the width of the arc $(b-c)$ for two different values of substrate thickness.

height increases, the rate of the resonant frequency reduction diminishes. It is also observed that the arc projection is more effective in reducing the resonant frequency for the thinner substrates. In other words, similar to the phenomenon that occurs for the cuboid ridge in a rectangular patch microstrip antenna [11], it is the ratio of the projection height to the substrate thickness $\left(h_{r} / h\right)$ that determines how effective the projection is.

The resonant frequency of the antenna reduces with the increases in the arc width. The variations of the resonant frequency as a function of the arc width are plotted for two values of the substrate thickness, $h=0.8 \mathrm{~mm}$ and $h=1.6 \mathrm{~mm}$, in Fig. 8 . The dielectric constant of the substrate is as before, $\varepsilon_{r 1}=2.2$, and the material used for the arc projection has the same characteristics as for the fabricated prototype. The outer and inner radii of the arc, i.e., $c$ and $b$, have the initial values of 17 and $4 \mathrm{~mm}$, respectively. For any step, the inner and outer radii reduce simultaneously by $0.2 \mathrm{~mm}$.

Simulation results show that similar to the cuboid ridge in the rectangular patch antennas, the dielectric constant of the material that created the projection has no significant contribution to the resonant frequency for short-height projections. However, its contribution increases as the height of the projection increases.

The proposed approach increases the design flexibility by introducing two extra parameters: projection height and arc width. Compared to other miniaturization techniques, the proposed approach has a simple design procedure and is relatively robust to the distortion of design parameters. The proposed approach is easy to fabricate and cost-efficient for production. Although the proposed antenna is a patch antenna, it should not necessarily be uniformly planar. Hence, the designer may efficiently use the volume dedicated to the antenna. However, such nonuniformity can be a disadvantage for some special applications that planarity is essential.

\section{CONCLUSION}

An approach to miniaturize the patch size of the circular patch microstrip antennas was presented. The proposed approach has been inspired from our earlier work, i.e., rectangular patch size reduction using a cuboid ridge. The presented approach is based on using a nonuniform substrate by introducing a dielectric semicircle arc projection on the antenna. The size of the patch is determined by the height of the projection as well as the width of the arc. The wider the arc and the taller the projection, the smaller the patch size. A prototype of the proposed antenna and a prototype of a conventional circular patch microstrip antenna were fabricated and measured. The two antennas were compared, and the simulation and measurement results were presented and discussed. It was shown that while a significant size reduction is achieved by the arc projection, no substantial degradation was observed in the antenna gain. Furthermore, introducing such an arc projection provides the designer with two more design parameters.

\section{ACKNOWLEDGMENT}

The supporting simulation files and raw measurement results for this letter can be accessed at https://figshare.com. The DOI for these data files is $10.17028 /$ rd.lboro. 3471827 .

\section{REFERENCES}

[1] C. A. Balanis, Antenna Theory: Analysis and Design, 3rd ed. Hoboken, NJ, USA: Wiley-Interscience, 2005.

[2] S. Pinhas and S. Shtrikman, "Comparison between computed and measured bandwidth of quarter-wave microstrip radiators," IEEE Trans. Antennas Propag., vol. 36, no. 11, pp. 1615-1616, Nov. 1988.

[3] R. Chair, K. F. Lee, and K. M. Luk, "Bandwidth and cross-polarization characteristics of quarter-wave shorted patch antennas," Microw. Opt. Technol. Lett., vol. 22, no. 2, pp. 101-103, 1999.

[4] R. Waterhouse, "Small microstrip patch antenna," Electron. Lett., vol. 31, no. 8, pp. 604-605, 1995.

[5] B. Lee and F. Harackiewicz, "Miniature microstrip antenna with a partially filled high-permittivity substrate," IEEE Trans. Antennas Propag., vol. 50, no. 8, pp. 1160-1162, Aug. 2002.

[6] T. Lo, C.-O. Ho, Y. Hwang, E. K. W. Lam, and B. Lee, "Miniature aperturecoupled microstrip antenna of very high permittivity," Electron. Lett., vol. 33, no. 1, pp. 9-10, 1997.

[7] K. Luk, R. Chair, and K.-F. Lee, "Small rectangular patch antenna," Electron. Lett., vol. 34, no. 25, pp. 2366-2367, 1998.

[8] S. Reed, L. Desclos, C. Terret, and S. Toutain, "Patch antenna size reduction by means of inductive slots," Microw. Opt. Technol. Lett., vol. 29, no. 2, pp. 79-81, 2001.

[9] X. Cheng, J. Wu, R. Blank, D. Senior, and Y.-K. Yoon, "An omnidirectional wrappable compact patch antenna for wireless endoscope applications," IEEE Antennas Wireless Propag. Lett., vol. 11, pp. 1667-1670, 2012.

[10] D. Wang, H. Wong, and C. H. Chan, "Small patch antennas incorporated with a substrate integrated irregular ground," IEEE Trans. Antennas Propag., vol. 60, no. 7, pp. 3096-3103, Jul. 2012.

[11] A. Motevasselian and W. Whittow, "Patch size reduction of rectangular microstrip antennas by means of a cuboid ridge," Microw., Antennas Propag., vol. 9, no. 15, pp. 1727-1732, 2015. 